\title{
Transient thermal regime trough the constitutive matrix applied to asynchronous electrical machine using the cell method
}

DOI: https://doi.org/10.1515/phys-2018-0090

Received July 9, 2018; accepted August 5, 2018

\section{Nomenclature}

Table 1: Symbols and units used

\begin{tabular}{|c|c|c|}
\hline Symbo & Name & unit \\
\hline$[A]$ & Scheme Crank-Nicolson's Matrix & $\mathbf{W} \cdot \mathrm{K}^{-1}$ \\
\hline$C, \tilde{C}$ & Primal and dual discrete curl operators. & - \\
\hline$C_{p}$ & Specific heat. & $\mathrm{J} \cdot \mathrm{kg}^{-1} \cdot \mathrm{K}^{-1}$ \\
\hline$D, \tilde{D}$ & Primal and dual discrete divergence operators. & - \\
\hline & Temperature variation. & ${ }^{\circ} \mathrm{C} \cdot \mathbf{s}^{-1}$ \\
\hline$G, \tilde{G}$ & Primal and dual discrete gradient operators. & - \\
\hline I & Electric current intensity. & A \\
\hline$L, \tilde{L}$ & Primal and dual cell edge longitude. & m \\
\hline$\left[M_{\lambda}\right]$ & $\begin{array}{l}\text { Constitutive matrix in Fourier's Law of Heat } \\
\text { Conduction }\end{array}$ & $\mathbf{W} \cdot \mathrm{K}^{-1}$ \\
\hline$\left[\boldsymbol{M}_{C_{p}}\right]$ & $\begin{array}{l}\text { Constitutive matrix in heat transition in } \\
\text { transitory state. }\end{array}$ & $J \cdot K^{-1}$ \\
\hline$\left[M_{\sigma}\right]$ & Constitutive matrix in electrical conduction. & $\Omega^{-1}$ \\
\hline$n_{k}$ & $\begin{array}{l}\text { Number of nodes of the cell. } \\
\text { Heat flow crossing a surface (included } q_{\lambda} \text { and }\end{array}$ & - \\
\hline$q$ & $\left.q_{c p}\right)$ & $\mathrm{J} / \mathrm{s}$ \\
\hline $\boldsymbol{S}, \tilde{\boldsymbol{S}}$ & Primal and dual surfaces. & $\mathrm{m}^{2}$ \\
\hline $\begin{array}{l}\text { l, } \mathrm{t}, \\
\Delta \mathrm{t}\end{array}$ & Time & $\mathbf{s}$ \\
\hline$V_{T}$ & Tetrahedron volume. & $m^{3}$ \\
\hline$v, \tilde{V}$ & Primal and dual volumes. & $\mathrm{m}^{3}$ \\
\hline$[v]$ & Electrical potential matrix & $\mathbf{v}$ \\
\hline$[W]$ & Heat generated during a process & $\mathbf{w}$ \\
\hline$\lambda_{k}$ & Volumetric thermal conductivity & $W \cdot K^{-1} \cdot m^{-1}$ \\
\hline$\rho$ & Material density & $\begin{array}{l}\mathrm{Kg} \cdot \\
\mathrm{m}^{-3}\end{array}$ \\
\hline $\boldsymbol{\tau}$ & Temperature & $\stackrel{\circ}{C}$ \\
\hline $\boldsymbol{\theta}$ & Scheme Crank-Nicolson's term: Euler implicit = 1 & - \\
\hline$\sigma$ & Electrical conductivity & $\Omega^{-1} \cdot m^{-1}$ \\
\hline
\end{tabular}

*Corresponding Author: Pablo Ignacio González-Domínguez: Departamento de Ingeniería Eléctrica, Instituto Universitario de Microelectrónica Aplicada, Universidad de Las Palmas de Gran Canaria, Canary Islands, Spain, E-mail: pablo.gonzalez@ulpgc.es José Miguel Monzón-Verona: Departamento de Ingeniería Eléctrica, Instituto Universitario de Microelectrónica Aplicada, Universidad de Las Palmas de Gran Canaria, Canary Islands, Spain, E-mail: josemiguel.monzon@ulpgc.es

Santiago García-Alonso : Departamento de Ingeniería Eléctrica, Instituto Universitario de Microelectrónica Aplicada, Universidad de Las Palmas de Gran Canaria, Canary Islands, Spain,

E-mail: santiago.garciaalonso@ulpgc.es

Abstract: In this paper, a new constitutive matrix for therconduction in transient thermal regime is developed tutive matrix defines through the cell method the behavior of solids when they are under a thermal potential. We have demonstrated that this matrix is equivalent to the electrical conduction constitutive matrix in steady state. We mulation. The final algebraic equation system is tailored directly without discretizing of the differential equations. This is an important advantage because we omit a complex differential formulation and the discretization of the

Keywords: Electric motors, finite element analysis, numerical analysis, numerical simulation, thermal analysis, electrical and thermal conduction

PACS: $84.50 .+d$, 02.70.Dh, 02.60.Lj, 05.45.Pq, 81.70.Pg, 72.15.Cz 
energy saving, etc. - we should pay special attention to a particular design problem, the electric machine thermal analysis.

Electromagnetic behavior and thermal analysis are strongly linked in electric machine analysis. There are excellent works that apply modern thermal analysis to electric machines [1, 2]. Among these models, those based on concentrated parameters are often used. These models are simple and they are easily implemented [3-5] but sometimes they are not accurate enough.

Other methods, such as finite element method (FEM), use both concentrated and distributed parameters. In addition, in the computational study of the convective effect [6] in fluid dynamics, FEM is commonly recognized as the most generalized method for electromagnetic-thermal coupled analysis [7], including electromagnetic-thermalmechanical couplings [8].

Other researchers use statistical experimental methods such as design of experiment (DOE) and response surface methodology (RSM) techniques for thermal power loss analysis [9].

Some authors investigate the experimental characterization of thermal properties, such as thermal conductivity and thermal effusivity and the measurement of these thermal properties [10-12].

In the present work, we use cell method (CM) as a numerical method [13] that is included in finite formulation methodology (FF). In FF, the physical laws governing the electromagnetic fields and the physical thermal phenomena are expressed in integral formulation. The final algebraic equation system is tailored directly without discretizing of the differential equations. The matrix of coefficients of this linear algebraic equation system is called global matrix. In previous works, we have presented some studies related to CM technologies $[14,15]$.

Clearly, this is an important advantage, because we omit a complex differential formulation and the discretization of the respective equations. Recently, CM has been used to analyze electromagnetic fields [14] and thermalelectromagnetic fields [16-18].

Our methodology can be generalized to other electric machines such as transformers, DC machines, synchronous machines and linear drives.

\section{$3 \mathrm{CM}$ and thermal conductivity}

The domain of interest is a thermal conduction region with a particular thermal conductivity $\left(\lambda_{k}\right)$. With CM, we determine the thermal distribution using a mesh composed of cells. Cells are geometry entities with their own physical properties, delimitated by nodes, edges and surfaces.

This domain is subdivided into a primal subspace and a dual subspace, with their respective discrete operators $[\mathrm{G}],[\mathrm{C}]$ and $[\mathrm{D}]$ for the primal cell, and $[\tilde{G}],[\tilde{C}]$ and $[\tilde{D}]$ for the dual cell. Primal cells are always associated to configuration field variables and dual cells are associated to source field variables.

Thus, the amount of heat (q) is associated with the dual cell volume $(\tilde{V})$ and the heat flow will flow through the dual cell faces $\left(\tilde{S}_{k}\right)$ that constrain the dual cell. However, the temperature $(\tau)$ is associated with the primal cell nodes $\left(n_{k}\right)$ because temperature is a configuration variable of the CM. Reducing one dimension, from 3-D to 2-D, will obviously decrease the cell dimension.

In CM, the Heat Transmission Equation for homogenous and isotropic media is expressed as [17-19]:

$$
[G]^{T}\left[M_{\lambda}\right][G][\tau]+\left[M_{C p}\right] \frac{d \tau}{d t}=[W]
$$

We observe that there are two constitutive matrices: $\left[M_{\lambda}\right]$ and $\left[M_{C p}\right]$. The thermal constitutive matrix $\left[M_{\lambda}\right]$ is the first term of (1) that belongs to Fourier's Law of Heat Conduction and the constitutive matrix $\left[M_{C p}\right]$ that indicates the body capacity to store heat energy, with temperature change expressed by $\frac{d \tau}{d t}$. The constitutive matrix $\left[M_{C p}\right]$, referred to an element (tetrahedron) of the mesh is:

$$
\left[M_{C p}\right]=\frac{\rho C_{p} V}{375}\left[\begin{array}{llll}
75 & 23 & 23 & 23 \\
23 & 75 & 23 & 23 \\
23 & 23 & 75 & 23 \\
23 & 23 & 23 & 75
\end{array}\right]
$$

To understand the meaning of the numerical matrix and the dividend, consult [20].

Equation (1) is a system of $n$ ordinary differential equations, where the unknowns are the temperature $\tau$ associated with the mesh nodes.

Based in (1) we have studied the temperature field produced by the electromagnetic fields generated in an asynchronous electrical machine, which is one of the most typical problems related to asynchronous electrical machines. Both constitutive matrices depend on the physical characteristics of the asynchronous machine constitutive materials. These characteristics are the thermal conductivity $(\lambda)$, the specific heat $\left(\mathrm{C}_{p}\right)$ and the material density $(\rho)$, and they are applied to the study of asynchronous machines. They are in connection with the capacity of the machine of storing the heat generated in a process. Vector $[W]$ in (1) represents the heat sources produced by Joule effect, magnetic hysteresis and eddy currents. Matrix $[G]$ stands for the primal discrete gradient. 
Fourier Heat Transmission equation in CM is studied in $[15,16]$. These references solve the problem by a transformation of the general absolute Cartesian cell coordinates to local normalized coordinates. This implies that absolute Cartesians coordinates of each cell have to be converted to normalized local coordinates. When these authors have finished their calculations, the output data are in local mode and they have to be assembled into the global matrix of the system. They have to convert the data into absolute coordinates, the global mode coordinates. This process of successive conversions is time-consuming so we have directly worked in general absolute Cartesian cell coordinates. When it is possible, other authors employ axial or planar symmetries in their models. In this way, the problem is reduced to a 2-D domain, see Figure 1 .

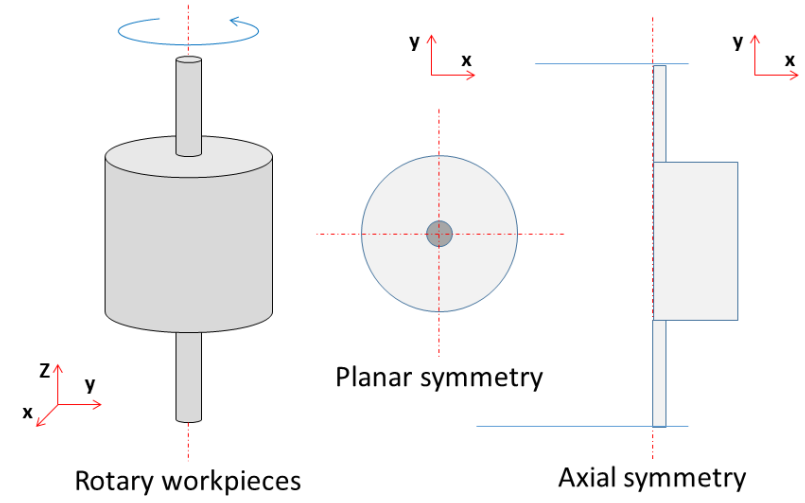

Figure 1: Planar and axial symmetry of a body of revolution

When there is an axisymmetric symmetry, the magnitude of the problem is also considerably reduced [13]. All the variables in the dual cell are projected to the primal cell. Here after, they perform all the calculations in the primal subspace.

However, it is not possible to use an axial or planar temperature distribution in asynchronous electric machines, as it can be seen in Figure 2. It is only possible in the squirrel cage rotor where the sources of heat are practically located in the volume that occupies. In this particular case, heat is generated in the shaft ends by mechanical friction and eddy currents, and the cage and the ferromagnetic core look like a cylindrical volume, see Figure 2a.

This approach cannot be applied to an asynchronous wound rotor machine, where the coil heads of the rotor do not have this symmetry. In addition, the electromagnetic and thermal effects are different in the heads of the coil and the cylindrical part of the rotor. The same behavior appears in the heads of the stator coil. See Figure 2b.

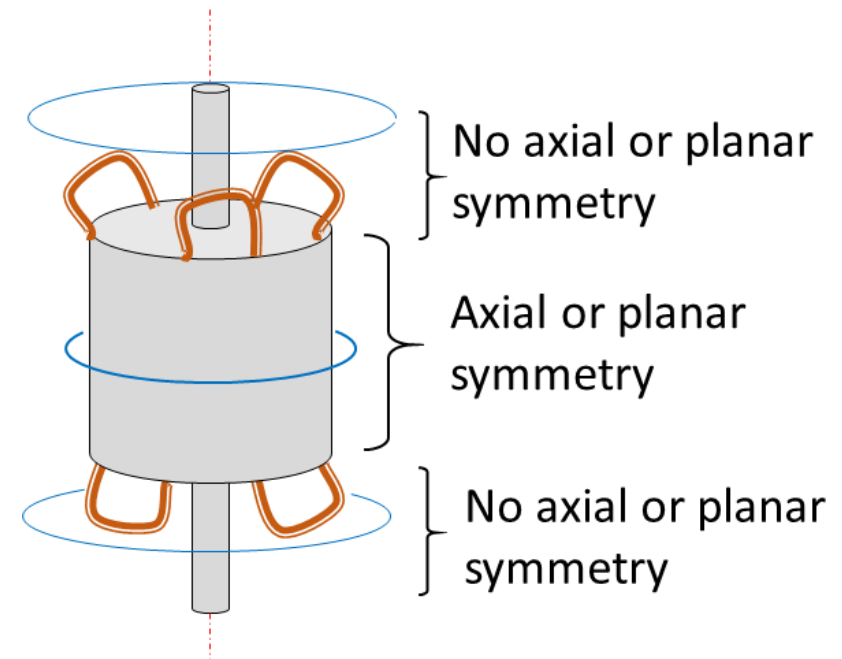

(a)

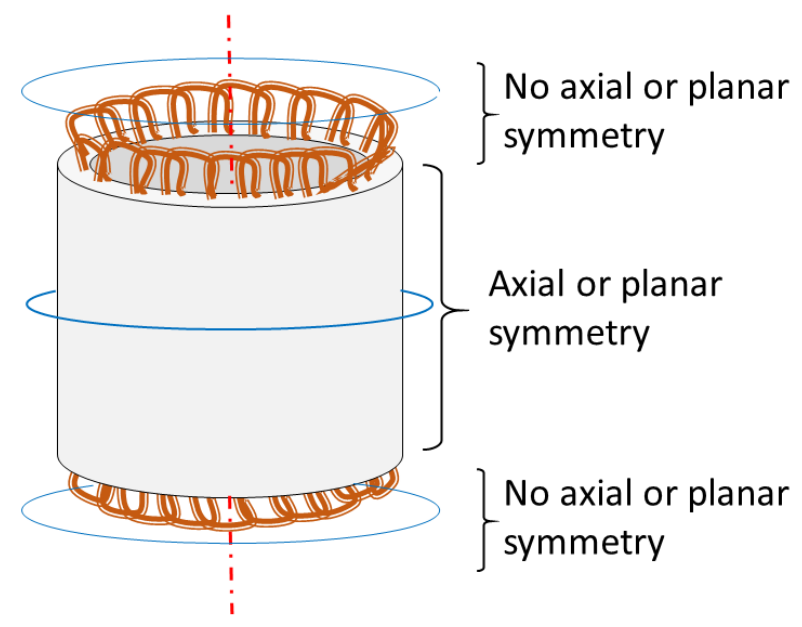

(b)

Figure 2: Symmetries of a rotor (a) and a stator (b) of an electric machine

The heat sources studied in this work are the Joule effect in wires and the magnetic hysteresis, and eddy currents in the ferromagnetic cores. The heat produced by mechanical friction is not studied.

Therefore, we have developed a new methodology to solve problems working with $\mathrm{CM}$ in a 3-D complex domain. Axial symmetries, planar symmetries, changes of coordinates and projection of the dual space into the primal space are not needed because we solve more general non-axisymmetric problems.

This leads to considerable savings in computational time and memory because we do not have to change coordinate definition from local to absolute and we do not have to translate the properties from an axisymmetric model to a 3-D model. 
The system of equations (1) is assembled volume to volume (tetrahedron). The equations that correspond to each tetrahedron are explained in Section 4.

\section{Thermal constitutive matrix}

The thermal constitutive matrix characterizes the behaviour of a domain with a thermal distribution and it is the key to solve a coupled electromagnetic thermal problem. This matrix is applied to each elements of the dual volume.

The first step to obtain this matrix is to separate the heat conduction equation in two terms, as can be seen in (1). The first term corresponds to the Fourier's Law of Heat Conduction applied to the rotor, the stator and the air gap, as can be seen in (3).

The second term represents the changes in internal energy, which depend on asynchronous machine materials, see (4). This equations system can to be used for global system (1) or for each element in the dual volume.

$$
\begin{gathered}
{[G]^{T}\left[M_{\lambda}\right][G][\tau]=\left[q_{\lambda}\right]} \\
{\left[M_{C p}\right] \frac{d \tau}{d t}=\left[q_{C p}\right]}
\end{gathered}
$$

It is equivalent to apply the dual divergence operator $[\tilde{D}]$ to the Fourier's Law of Heat Conduction in (5) and to the electrical current conduction law in (6). In a temperature field, a non-zero divergence indicates if we are working with a positive or a negative heat source.

However, when the divergence is zero, the materials conduct heat without sources or sinks.

$$
\begin{gathered}
{[\tilde{D}]\left\{-\left[M_{\lambda}\right][G][\tau]\right\}=[\tilde{D}]\left[q_{\lambda}\right]} \\
{[\tilde{D}]\left\{-\left[M_{\sigma}\right][G][v]\right\}=[\tilde{D}][I]}
\end{gathered}
$$

But, if we operate with the dual divergence operator and we seek its equivalent in the primal cell [10], then (5) and (6) can be expressed as (7) and (8), where $[G]$ is the primal gradient operator, with changes in the sign and the operator.

$$
\begin{gathered}
-[G]^{T}\left\{-\left[M_{\lambda}\right][G][\tau]\right\}=-[G]^{T}\left[q_{\lambda}\right] \\
-[G]^{T}\left\{-\left[M_{\sigma}\right][G][v]\right\}=-[G]^{T}[I]
\end{gathered}
$$

Therefore, we can conclude that the thermal and electrical conductivity constitutive matrix are equivalent. That is,

$$
\left[M_{\lambda}\right] \Leftrightarrow\left[M_{\sigma}\right]
$$

Therefore, there is an equivalence between electric conductivity and thermal conductivity in CM and we take the term proposed in [14]:

$$
\left[M_{\sigma}\right]=\frac{\sigma}{V_{T}}[S]_{6 \times 6}
$$

We set the equivalence proposed in (10),

$$
\frac{\lambda}{V_{T}}[S]_{6 \times 6} \Leftrightarrow \frac{\sigma}{V_{T}}[S]_{6 \times 6}
$$

Where matrix $[S]$ is defined according to (11), following Specogna and F. Trevisan [16], by

$$
[S]_{6 \times 6}=\left[\begin{array}{cccccc}
\overrightarrow{\tilde{S}}_{0} \overrightarrow{\tilde{S}}_{0} & \cdots & \ldots & \ldots & \ldots & \overrightarrow{\tilde{S}}_{0} \overrightarrow{\tilde{S}}_{5} \\
\vdots & \ddots & & & & \vdots \\
\vdots & & \overrightarrow{\tilde{S}}_{2} \overrightarrow{\tilde{S}}_{2} & & & \vdots \\
\vdots & & & \ddots & & \vdots \\
\vdots & & & & \ddots & \vdots \\
\overrightarrow{\tilde{S}}_{5} \overrightarrow{\tilde{S}}_{0} & \cdots & \ldots & \ldots & \ldots & \overrightarrow{\tilde{S}}_{5} \overrightarrow{\tilde{S}}_{5}
\end{array}\right]_{6 \times 6}
$$

where $\overrightarrow{\widetilde{S}}_{i} \overrightarrow{\tilde{S}}_{j}$ is the dot product of the vectors that define the dual faces of the cell and $V_{T}$ is the volume of tetrahedron. The advantage of the proposed method is that it is necessary to calculate matrix [S] only once instead of the usual calculations that make two evaluations, one for the electrical problem and one for the thermal problem, see Figure 3. $\underline{2 \mathrm{D}}$
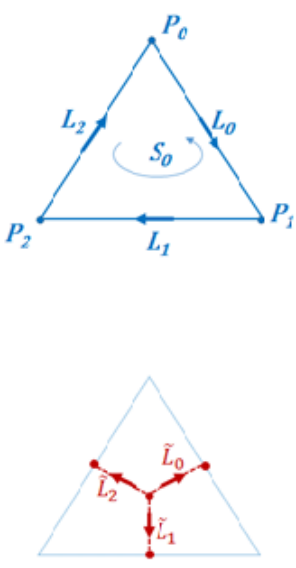

$\underline{3 D}$

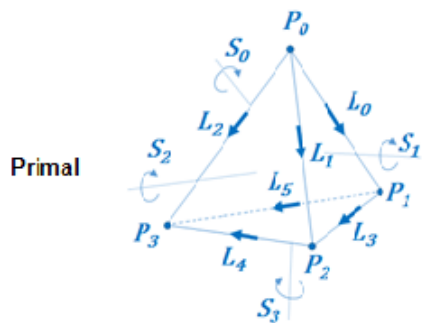

Dual

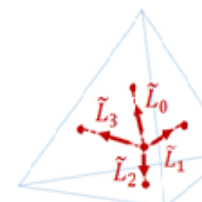

Figure 3: Cells in 2-D and 3-D

In one of our previous works [19] we have demonstrated that FF-CC methodology is valid for calculating 
processes of heat transmission in steady state. In the present work we demonstrate that $\mathrm{CM}$ is also a valid method when it is applied in heat transmission in transient regime.

\section{Numerical results}

In this section we will check that FF-CM is valid to analyse a transient electro-thermic process and that the proposed matrix $\left[M_{\lambda}\right]$ is also valid in transient regime.

Our model consists on a cube that covers a conductor rod. We have called this prototype 1 CUBO as it can be seen in Figure 4. In the model 1CUBO, the inner prism represents a segment of the rotor winding or the stator winding in an electric machine. This wire will get hot by the effect of electric currents. The outer prism is surrounded by air.

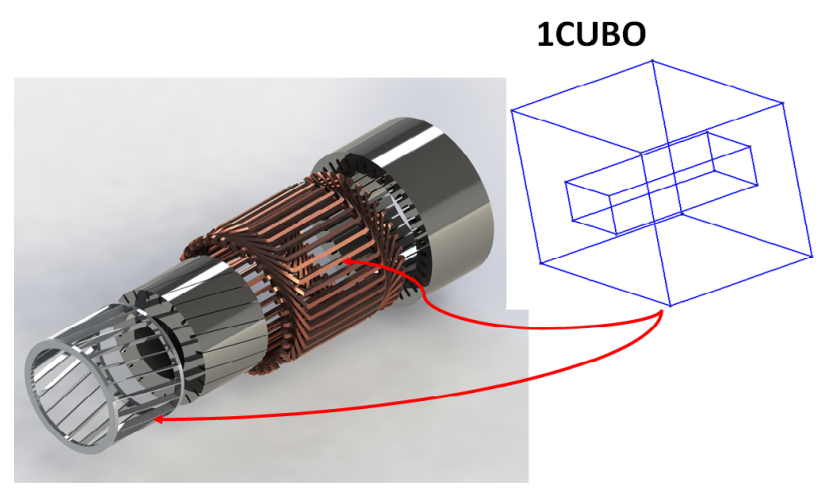

Figure 4: Model 1CUBO

Both cube and rod are crossed by a line where temperature has been measured, see Figure 5 a.

The experiment consists on applying an electrical potential difference to the element 1CUBO in Figure 4. This difference in electrical potential is $0.1 \mathrm{~V}$. An electric current is created. This electric current generates heat represented by [W]. This matrix represents the heat sources with their respective thermal potentials in the primal nodes - temperatures. We use the interval $\mathrm{T}=[0.00,10.00]$ seconds, with increments of 0.1 seconds. To realise the experiment with FF-CM we have made a parametric cut that crosses the whole volume of the envelope (air) and conductor rod (cupper) of the model 1CUBO. This parametric cut line is shown in Figure 5a, b and c.

To contrast the obtained results, a surface has been designed which is equivalent to the transversal section where the experiment has been carried out with FF-CM. This sec- tion contains a parametric cut identical to that previously explained.

The same conditions have been simulated with Getdp [21] in 2D and the obtained results have been used as a comparison pattern.

The equation that uses FF-CM (1), with a scheme Crank-Nicolson, is the following,

$$
\begin{aligned}
\left(\frac{1}{\Delta t}\left[M_{C p}\right]+\theta[A]\right) \tau^{n+1}= & \left(\frac{1}{\Delta t}\left[M_{C p}\right]-(1-\theta)[A]\right) \tau^{n} \\
& +(1-\theta)\left[W_{i}^{n}\right]+\theta\left[W_{i}^{n+1}\right]
\end{aligned}
$$

where

$$
[A]=[G]^{T}\left[M_{\lambda}\right][G]
$$

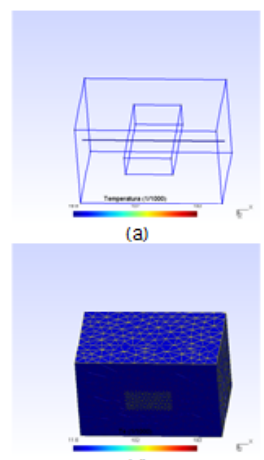

(d)

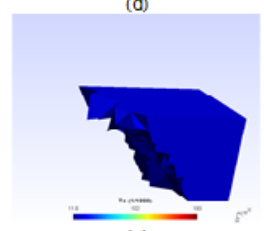

(g)

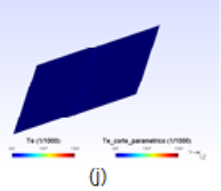

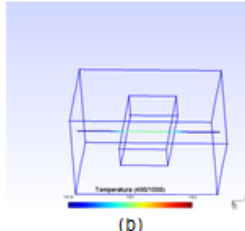

(b)

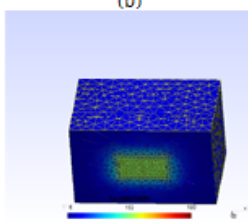

(e)

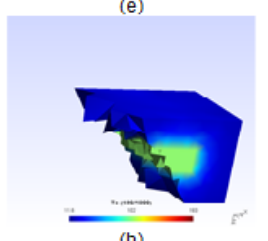

(h)

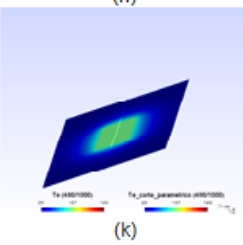

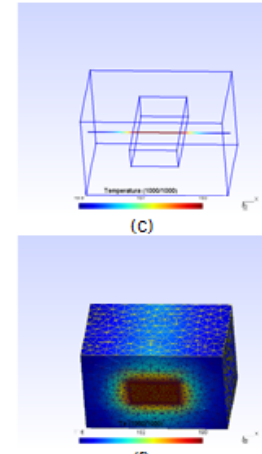

(f)

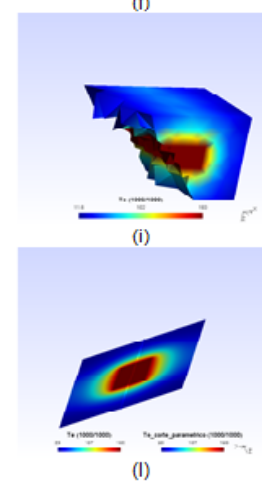

Figure 5: Electro-thermal transient state in 1CUBO model. The first column shows the result for $\mathrm{t}=0.01 \mathrm{~s}$, the central column shows the result for $t=5.07 \mathrm{~s}$ and the third column shows the result for $t=9.91 \mathrm{~s}$. The first row shows the parametric line, the second one shows in 3D the 1CUBO model, the third one represents the inner of the model and finally, the fourth ones shows a 2D model.

The data of the experiment are the following,

Boundary conditions are: the cupper domain $\Omega_{\mathrm{Cu}}$, which is an electric and thermal conductor, air domain $\Omega_{\text {air }}$ is a thermal conductor and the envelope domain $\Omega_{\text {envelope }}$ is an electric and thermal insulator. 
Table 2: Physical and numeric parameters for the simulations

\begin{tabular}{|c|c|c|c|c|c|c|}
\hline$V:$ & 0,1 & v & $\mathbf{x x}$ & $M_{\text {air }}:$ & $B C$ & kg \\
\hline Ig: & 0,0 & $\mathbf{A}$ & & $C p_{\text {air }}:$ & 1012 & Ud2 \\
\hline$t_{0}:$ & 0,0 & $\mathrm{~s}$ & & $T_{0 \_ \text {air }}:$ & 20 & ${ }^{\circ} \mathrm{C}$ \\
\hline$t_{f}:$ & 10,0 & $\mathbf{s}$ & & $M_{c u}:$ & $B C$ & kg \\
\hline$\theta:$ & 1 & IE & & $C p_{c u}:$ & 387 & Ud2 \\
\hline$\Delta t:$ & 0,01 & $\mathbf{s}$ & & $\lambda_{c u}:$ & 372,10 & Ud3 \\
\hline$\overline{\gamma_{a i r}:}$ & 1,25184 & Ud1 & & $\gamma_{c_{u}}:$ & 8940 & Ud1 \\
\hline$\overline{\lambda_{\text {air }}:}$ & 0,024 & Ud4 & & $T_{0 \_C u}:$ & 20 & ${ }^{\circ} \mathrm{C}$ \\
\hline$\overline{\sigma_{\text {air }}:}$ & $\because-$ & NA & & $\sigma_{C u}:$ & $58 \cdot 10^{6}$ & $\mathrm{~S} / \mathrm{m}$ \\
\hline
\end{tabular}

Ud1: $\mathrm{Kg} / \mathrm{m}^{3}, 25^{\circ} \mathrm{C}, 1$ ATA; Ud2: $\mathrm{J} \cdot \mathrm{kg}^{-1} \cdot \mathrm{K}^{-1}, 25^{\circ} \mathrm{C}, 1$ ATA; Ud3: $\mathbf{W} \cdot \mathrm{m}^{-1} \mathrm{~K}^{-1}$; Ud4: $\mathbf{W} \cdot \mathrm{m}^{1} \mathrm{~K}^{-1}$; IE: Implicit Euler; NA: Not applicable; BC: By calculation.

Initial conditions are: $\tau_{0 \_C u}: \quad 20.00{ }^{\circ} \mathrm{C}$ y $\tau_{0 \_ \text {air }}$ : $20.00{ }^{\circ} \mathrm{C}$.

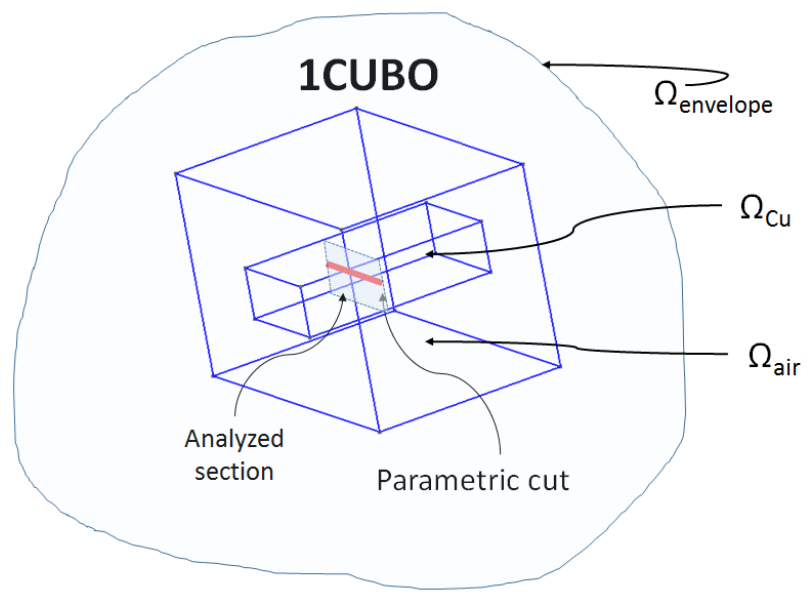

Figure 6: Domains for the model 1CUBO

The time step used is $\Delta \mathrm{t}=0.01$ seconds, as can be seen in Table 2. We have taken thermal data in the parametric cutline in three different arbitrary instants of time [0.01, 5.07, 9.91] seconds, as it is shown in Figure 7. With these three instants of time, we demonstrate that the three numerical methods are coincident. Therefore, our method 3D FF-CM is validated, see Figure 7.

We have developed three models to analyse the prototype 1CUBO.

The first model is developed with FF-CM in 3D. The second one is calculated with Getdp in 2D. And finally, the third model is worked out with Getdp in 3D.

We have compared the results obtained with these three methods. They have been compared in pairs to determine the errors committed, as it is shown in Table 3.

We have used 2D and 3D Getdp as a reference for the estimation of the result accuracy in FF-CM. In this way, we

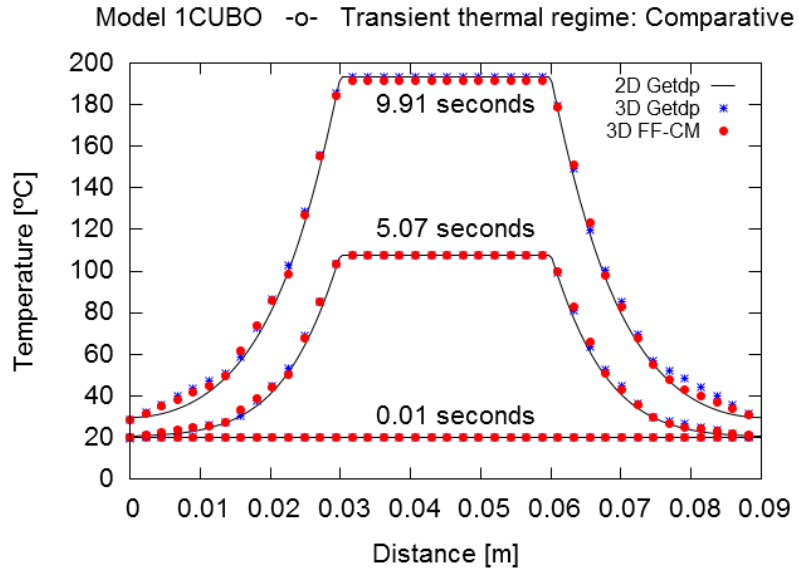

Figure 7: Thermal transient using constitutive thermal matrix $\left[M_{\lambda}\right]$

Table 3: Comparatives between different numerical experiments

\begin{tabular}{ll}
\hline C1: & FF-CM vs 2D Getdp in 0.01 s \\
\hline C2: & FF-CM vs 2D Getdp in $5.07 \mathrm{~s}$ \\
\hline C3: & FF-CM vs 2D Getdp in 9.91 s \\
\hline C4: & FF-CM vs 3D Getdp in 0.01 s \\
\hline C5: & FF-CM vs 3D Getdp in 5.07 s \\
\hline C6: & FF-CM vs 3D Getdp in 9.91 s \\
\hline
\end{tabular}

compare the results obtained through FF-CM in 3D and 2D with those obtained with Getdp. Getdp 2D is more accurate than Getdp3D in axisymmetric problems because you can use denser meshes with the same number of finite elements.

We have verified the accuracy of the results obtained though the new constitutive matrix of FF-CM by three different methods.

\subsection{First verification}

The statistics of error or metrics are models that allow the development of fit criteria to estimate the error introduced by experimentation or simulation compared to specific reference functions. That is to say, they allow to quantify the error committed with regard the data of reference.

The statistics used in the validation of the models are the following, $R^{2}$, Determination Coefficient, see [22-24]. MSE: Mean Square Error, see [22-28] . RMSE: Root Mean Square Error, see [28-30]. RMSPE: Root M. S. Perceptual Error, see [31]. MAE: Mean Absolute Error, see [25-30]. MAEP: Mean Absolute Percentage Error, see [31]. PBIAS, see [22, 25, 32, 33], NSEF: Modelling Efficiency Nash \& Sutcliffe, see [25, 27, 34].U1: Theil Inequality Coefficient, see $[26,33,35] . \mathrm{U}^{M}$ : Bias Proportion or difference between 
Table 4: Comparative metrics of Table 3 cases

\begin{tabular}{|c|c|c|c|c|c|c|}
\hline Com & 1 & $\mathrm{C} 2$ & C3 & C4 & C5 & C6 \\
\hline $\begin{array}{l}\mathrm{R}^{2}[0,+1] \\
\text { Optimum: +1 }\end{array}$ & 0,857 & 0,998 & 0,999 & 0,952 & 0,999 & ,999 \\
\hline $\begin{array}{l}\text { MSE }[0,+\infty] \\
\text { Optimum: } 0\end{array}$ & 0,001 & 3,857 & 8,693 & 0,001 & 0,878 & 4,643 \\
\hline $\mathrm{MSE}\left[{ }^{\circ} \mathrm{C}\right]$ & 033 & 1,96 & 2,948 & 0,021 & 0,937 & .155 \\
\hline $\begin{array}{l}\text { RMSPE }[-1,+1] \\
\text { Optimum: } 0\end{array}$ & 0,002 & 0,032 & 0,026 & 0,001 & 0,015 & 0,019 \\
\hline MAE $\left[{ }^{\circ} \mathrm{C}\right]$ & 012 & 1,231 & 2,493 & 0,011 & 0,590 &, 874 \\
\hline $\begin{array}{l}\text { AAEP }[-1,+1] \\
\text { ptimum: } 0\end{array}$ & 001 & 0,020 & 0,022 & 0,001 & 0,009 & 0,016 \\
\hline pMI & j93 & 3,133 & 3,487 & 0,042 & 1,489 & 2,477 \\
\hline $\begin{array}{l}\text { BIA } \\
\text { ptir }\end{array}$ & 1 & 19 & 2 & 0,000 & 0,000 & 0,012 \\
\hline ptir & 833 & 0,997 & 0,998 & 0,940 & 0,999 & ,999 \\
\hline tir & 001 & 0,013 & 0,011 & 0,001 & 0,006 &, 008 \\
\hline ptin & 0,109 & 0,362 & 0,211 & 0,178 & 0,001 & 0,418 \\
\hline$\frac{\text { Optin }}{\mathrm{UC} \text { de }}$ & 0,000 & 0,017 & 0,271 & 0,067 & 0,001 &, 004 \\
\hline $\begin{array}{l}\text { Optin } \\
\text { Willm }\end{array}$ & 0,891 & 0,621 & 0,518 & 0,754 & 0,999 & 0,577 \\
\hline Optin & 0,958 & 0,999 & 0,999 & 0,984 & 0,999 & 0,999 \\
\hline Optir & 0,835 & 0,997 & 0,998 & 0,940 & 0,999 & 0,999 \\
\hline$\frac{\text { Optimum: } 1}{C|-\infty,+\infty|}$ & 0,993 & 1,013 & 1,046 & 1,127 & 0,999 & 0,995 \\
\hline Optimum: 0 & 01 & 0,019 & 0,022 & 0,001 & 0,009 & 0,016 \\
\hline
\end{tabular}

means (systematical error), see [26]. $\mathrm{U}^{S}$ : Variance Proportion (systematical error), see [26]. $\mathrm{U}^{C}$ : Covariance Proportion (not systematical error), see [26]. d: d-Willmott Index, see [36]. MEF: Modelling Efficiency, see [37]. CD: Determination Coefficient of Modelling, see [37]. C: Error Coefficient of Modelling, see [37].

Table 4 shows the metrics applied to the comparisons stablished in Table 3 and points out the validity ranges of each indicator.

In all the matrix shown in Table 4 we can see that all of them are near the optimum. MAE, which is given by the true value of the measured magnitude, in Celsius degrees, indicates small errors, under $3{ }^{\circ} \mathrm{C}$.

This first verification confirms the validity of FF-CM applied to transient thermic analysis.

\subsection{Second verification}

The second verification it is based in the Error Gauss's law. This law stablishes that null error should be the mean of the distribution, grouping the rest around this value. We have developed as an example two graphics for comparing C1and C6, (see Figure 8).

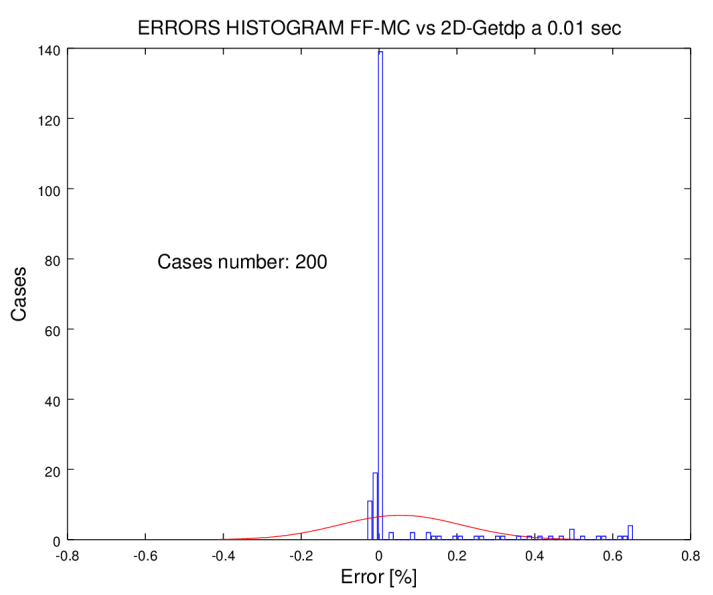

(a)

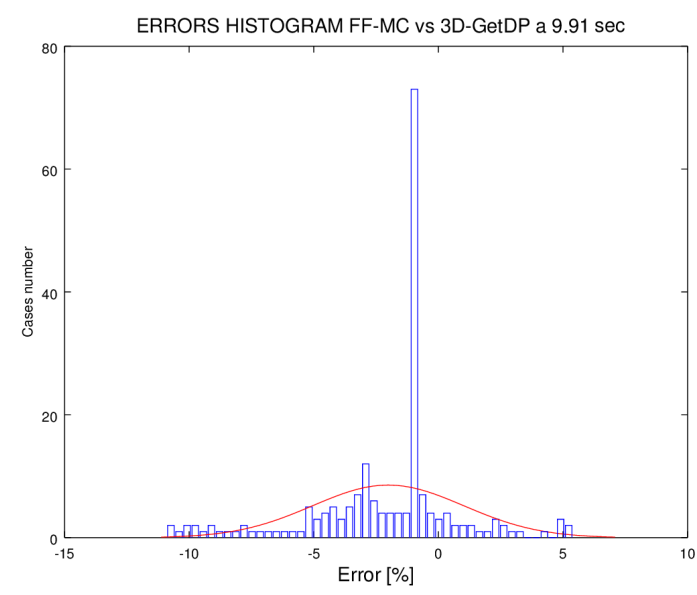

(b)

Figure 8: Errors distributions for the different comparatives. (a) Error distribution for comparative C1 (Table 3). (b) Error distribution for comparative C6 (Table 3).

It can be seen that null error is the most frequent error, and the other errors are distributed in a normal shape following Gauss`s law.

The distortion of the distributions from a normal distribution is due mainly to the fact that the cutline does not coincide exactly with the nodes of the mesh, see Figure 6.

This second verification, validates graphically the use of FF-CM in the transient thermal analysis, because the committed errors are very small. 


\subsection{Third verification}

In Figure 9a, 9b and 9c we realize a comparative analysis of errors in 3D FF-CM and 3D Getdp, taking as a reference 2D Getdp, in the three time instants considered. The errors in most of the cases are lower for FF-CM.

The distribution of the error is not symmetric in the cutline because the mesh of the model is not totally symmetrical. The asymmetric of these errors appears in Figure 9 because they are relative errors, while in Figure 7 they are absolute errors and hence they are very small.

This is due to the fact that while the thermal mass matrix in FFM has a condition number of 5 , the thermal mass matrix in FF-CM has a condition number of 2.7692. This implies that FF-CM is more accurate in the transient thermal analysis than FEM

The mass matrix for FEM that proposes Driesen [38] is

$$
M_{F E M}=\left[\begin{array}{llll}
2 & 1 & 1 & 1 \\
1 & 2 & 1 & 1 \\
1 & 1 & 2 & 1 \\
1 & 1 & 1 & 2
\end{array}\right]
$$

The mass matrix proposed by Bullo and which we use in this work for FF-CM is [18]

$$
M_{F F C M}=\left[\begin{array}{llll}
75 & 23 & 23 & 23 \\
23 & 75 & 23 & 23 \\
23 & 23 & 75 & 23 \\
23 & 23 & 23 & 75
\end{array}\right]
$$

\section{Conclusions}

We have demonstrated that FF-CM and $\left[M_{\lambda}\right]$ matrix are valid both in stationary and transient thermal regime. It should be emphasized that this matrix has been used only for electromagnetic problems.

In the same way, we have demonstrated that FF with the Cell Method and the new proposed matrix $\left[M_{\lambda}\right]$ can surpass the accuracy of FEM, in transient thermal analysis, due to the improvement in the condition number of the constitutive mass matrix.

Acknowledgment: This work was funded by project SURF (TEC2014-60527-C2-1-R) of the Spanish Ministry of Economy and Competitiveness.

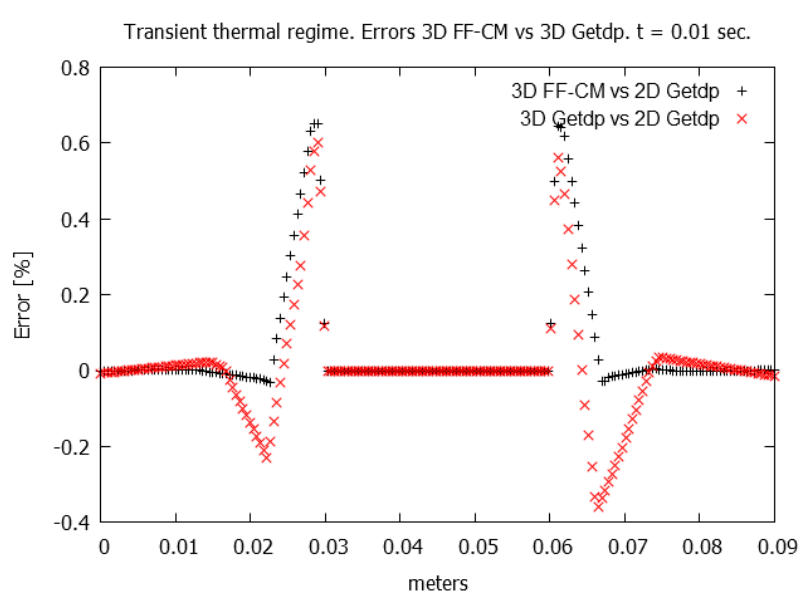

(a)

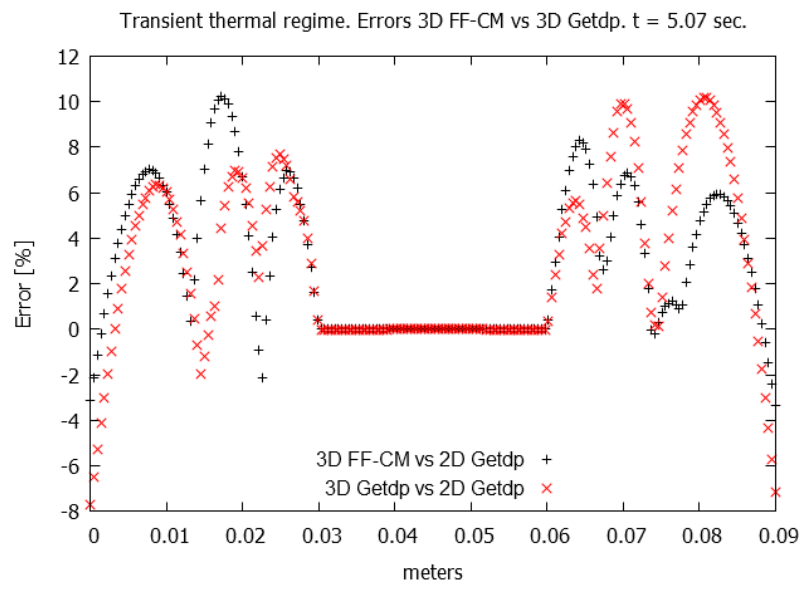

(b)

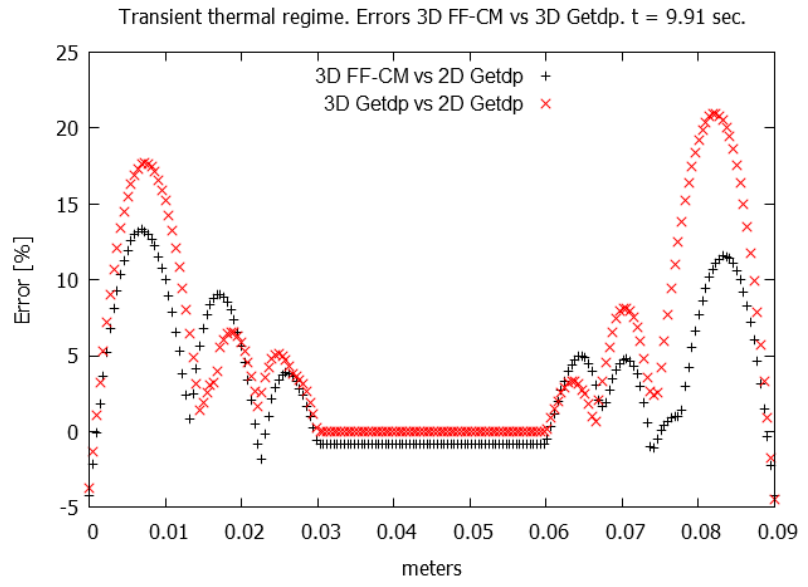

(c)

Figure 9: Errors between 3D models FF-CM and Getdp vs. 2D model Getdp 


\section{References}

[1] Boglietti A., Cavagnino A., Staton D., Shanel M., Mueller M., Mejuto C., Evolution and Modern Approaches for Thermal Analysis of Electrical Machines, IEEE Trans. Industrial Electronics, 2009, 56(3), 871-882.

[2] Popescu M., Staton D., Boglietti A., Cavagnino A., Hawkins D., Goss J., Modern heat extraction systems for electrical machines - A review, Electrical Machines Design, Control and Diagnosis (WEMDCD), 2015 IEEE Workshop, Torino, 2015, 289296.

[3] Mezani S., Takorabet N., Laporte B., A combined electromagnetic and thermal analysis of induction motors, IEEE Trans. Magnetics, 2005, 41(5), 1572-1575.

[4] Popova L., Nerg J., Pyrhönen J., Combined Electromagnetic and thermal design platform for totally enclosed induction machines, Diagnostics for Electric Machines, Power Electronics \& Drives (SDEMPED), 2011 IEEE International Symposium on, Bologna, 2011, 153-158.

[5] Boglietti A., Cavagnino A., Popescu M., Staton D., Thermal Model and Analysis of Wound-Rotor Induction Machine, IEEE Trans. Industry Applications, 2013, 49(5), 2078-2085.

[6] Nategh S., Huang Z., Krings A., Wallmark O., Leksell M., Thermal Modeling of Directly Cooled Electric Machines Using Lumped Parameter and Limited CFD Analysis, IEEE Trans. Energy Conversion, 2013, 28(4), 979-990.

[7] Jiang W., Jahns T.M., Coupled Electromagnetic-Thermal Analysis of Electric Machines Including Transient Operation Based on Finite-Element Techniques, IEEE Trans. Industry Applications, 2015, 51(2), 1880-1889.

[8] Sun X., Cheng M., Zhu S., Zhang J., Coupled ElectromagneticThermal-Mechanical Analysis for Accurate Prediction of DualMechanical-Port Machine Performance, IEEE Trans. Industry Applications, 2012, 48(6), 2240-2248.

[9] Seong K.H., Hwang J., Shim J., Cho H.W., Investigation of Temperature Rise in an Induction Motor Considering the Effect of Loading, IEEE Trans. Magnetics, 2014, 50(11), 1-4.

[10] Lin Qiu, Hanying Zou, Dawei Tang, Dongsheng Wen, Yanhui Feng, Xinxin Zhang, Inhomogeneity in pore size appreciably lowering thermal conductivity for porous thermal insulators, Applied Thermal Engineering, 2018, 130, 1004-1011.

[11] Qiu L., Zheng X.H., Zhu J., Tang D.W., Non-destructive measurement of thermal effusivity of a solid and liquid using a freestanding serpentine sensor-based $3 \omega$ technique, Review of Scientific Instruments, Rev. Sci. Instrum., 2011, 82(8), 086110.

[12] Qiu L., Zheng X.H., Yue P., Zhu J., Tang D.W., Dong Y.J., Peng Y.L., Adaptable thermal conductivity characterization of microporous membranes based on freestanding sensor-based $3 \omega$ technique, Int. J. Therm. Sci., 2015, 89, 185-192.

[13] Tonti E., A direct discrete formulation of field laws: The cell method, CMES- Computer Modeling in Engineering and Sciences, 2001, 2(2), 237-258.

[14] González-Domínguez P.I., Monzón-Verona J.M., Contribution to the improvement of the computing time for G, C, D matrix in the Cell Method, EUROGEN 2013, 2013, 201-203.

[15] Monzón-Verona J.M., Simón-Rodríguez L., GonzálezDomínguez P.I., Global Equation of Cell Method and Modified Nodal Method of Electromagnetic and Circuital Problems, ISEF
2011, Int. Symposium on Electromagnetic Fields in Mechatronics, Electrical and Electronic Engineering, Funchal, 2011, Madeira.

[16] Specogna R., Trevisan F., Discrete constitutive equations in A-Chi geometric eddy-current formulation, IEEE Trans. Magnetics, 2005, 41(4), 1259-1263.

[17] Bullo M., D’Ambrosio V., Dughiero F., Guarnieri M., Coupled electrical and thermal transient conduction problems with a quadratic interpolation cell method approach, IEEE Trans. Magnetics, 2006, 42(4), 1003-1006.

[18] Bullo M., D’Ambrosio V., Dughiero F., Guarnieri M., A 3D Cell Method Formulation for Coupled Electric and Thermal Problems, IEEE Trans. Magnetics, 2007, 43,(4), 1197-1200.

[19] GonzálezDomínguez P. I., Monzón-Verona J. M., Simón Rodríguez L., de Pablo Sánchez A., Thermal constitutive matrix applied to asynchronous electrical machine using the cell method, Open Physics, 2018, 16, 27-30.

[20] Voitovich T.V., Vandewalle S., Exact integration formulas for the finite volume element method on simplicial meshes. Numerical Methods for Partial Differential Equations, 2007, 23(5), 1059-1082.

[21] Geuzaine C., GetDP: A general finite-element solver for the de Rham complex, In Proceedings of the Sixth International Congress on Industrial Applied Mathematics (ICIAM07) and GAMM Annual Meeting, Zürich, Switzerland, 16-20 July 2007.

[22] Tedeschi L.O., Assessment of the adequacy of mathematical models, Agricultural Systems, 2006, 89(2), 225-247.

[23] Martínez Rodríguez E., Errores frecuentes en la interpretación del coeficiente de determinación lineal, Anuario jurídico y económico escurialense, 2005, 38, 315-331.

[24] Piñeiro G., Perelman S., Guerschman J.P., Paruelo J.M., How to evaluate models: observed vs. predicted or predicted vs. observed? Ecological Modelling, 2008, 216(3), 316-322.

[25] Moriasi D. et al., Model evaluation guidelines for systematic quantification of accuracy in watershed simulations, Trans. Asabe, 2007, 50(3), 885-900.

[26] Fullerton Jr T.M., Novela G. et al., Metropolitan Maquiladora Econometric Forecast Accuracy, Romanian Journal of Economic Forecasting, 2010, 13(3), 124-140.

[27] Gupta H.V., Kling H., Yilmaz K.K., Martinez G.F., Decomposition of the mean squared error and performance criteria: Implications for improving hydrological modelling, J. Hydrology, 2009, 377(1), 80-91.

[28] Jolliffe I.T., Stephenson D.B., Forecast verification: a practitioner's guide in atmospheric science, 2012, First ed. Sussex, UK, John Wiley \& Sons

[29] Chai T., Draxler R.R., Root mean square error (RMSE) or mean absolute error (MAE)? Arguments against avoiding RMSE in the literature, Geoscientific Model Development, 2014, 7(3), 1247-1250.

[30] Willmott C.J., Matsuura K., Advantages of the mean absolute error (MAE) over the root mean square error (RMSE) in assessing average model performance, Climate research, 2005, 30(1), 79.

[31] Hyndman R.J., Koehler A.B., Another look at measures of forecast accuracy, Int. J. Forecasting, 2006, 22(4), 679-688.

[32] Sanabria J., García J., Lhomme J.P., Calibración y validación de modelos de pronóstico de heladas en el valle del Mantaro, ECIPERU, 2006, 18. 
[33] Leuthold R.M., On the Use of Theil's Inequality Coefficients, Amer. J. Agricult. Econom., 1975, 57(2), 344-346.

[34] Mathevet T., Michel C., Andreassian V., Perrin C., A bounded version of the Nash-Sutcliffe criterion for better model assessment on large sets of basins, IAHS PUBLICATION, 2006, 307, 211.

[35] Bliemel F., Theil's Forecast Accuracy Coefficient: A Clarification, Journal of Marketing Research, 1973, 10(4), 444-446.

[36] Willmott C., Robesonb S., Matsuuraa K., Short Communication: A Refined Index of Model Performance, Int. J. Climatology, 2012, 32(13), 2088-2094.
[37] Medina-Peralta S., Vargas-Villamil L., Navarro-Alberto J., Canul-Pech C., Peraza-Romero S., Comparación de medidas de desviación para validar modelos sin sesgo, sesgo constante o proporcional, Universidad y ciencia, 2010, 12, 26, 255-263.

[38] Driesen J., Coupled Electromagnetic-Thermal Problems in Electrical Energy Transducers, Phd. Thesis, Katholieke Universiteit Leuven. Facluteit Toegepaste Wetenschappen. Departament Electrotechniek (ESAT). Afdeling Elecrische Energie (ELEN), 2000, Leuven 